\title{
Anesthetic Management of Hepatitis C Positive Patient with Portal Hypertension for Cesarean Section - A Case Report
}

\author{
Rachna Wadhwa, Geetanjali Chilkoti, Sunny Kumar and Ashok Kumar Saxena \\ Department of Anesthesiology and Critical care, University College of Medical Sciences and Guru Teg \\ Bahadur Hospital, Shahdara, Delhi, India
}

Correspondence should be addressed to: Rachna Wadhwa; drrachnawadhwa@gmail.com

Received Date: 30 November 2013; Accepted Date: 18 March 2014; Published Date: 26 April 2014

Academic Editor: Ishag I. Adam

Copyright @ 2014 Rachna Wadhwa, Geetanjali Chilkoti, Sunny Kumar and Ashok Kumar Saxena.

Distributed under Creative Commons CC-BY 3.0

\begin{abstract}
Viral hepatitis during pregnancy is associated with increased maternal and fetal mortality. Hepatitis C associated portal hypertension induced bleeding esophageal varices in pregnancy is a rare presentation. We report a case of successful perioperative management of a patient with history of hepatitis $\mathrm{C}$, mild portal hypertension and bleeding esophageal varices planned for cesarean section in view of breech presentation. The presence of coagulopathy, possibility of variceal rupture and need of transfusion of blood and blood products calls for comprehensive care and optimal management throughout the antenatal period. For such patients, regional anesthesia, if possible, is preferred over general anesthesia for emergency LSCS.
\end{abstract}

Keywords: Portal hypertension, Hepatitis C, Bleeding esophageal varices, Caesarean Section.

\section{Introduction}

Viral hepatitis during pregnancy predisposes to various maternal and fetal complications like jaundice, coagulopathy, hepatic failure, bleeding from esophageal varices, hepatorenal syndrome, spontaneous abortions and premature labour. In the review by Tan et al (2008), maternal mortality is generally attributed to massive bleeding from esophageal varices with incidence varying from $18-50 \%$. In the present case, we discuss the anesthetic management in patient with viral hepatitis and bleeding esophageal varices for lower segment caesarean section (LSCS).

\section{Case Report}

A 26 yr old primigravida, weighing 58kgs, with hepatitis $C$ and portal hypertension was scheduled for elective LSCS in view of breech presentation of fetus. Past history revealed few bouts of hemetemesis at 20 weeks of gestation with no prior history of fever, malena or altered mental status. Patient was hospitalized and investigated. The liver function tests (LFT) reported total

Cite this Article as: Rachna Wadhwa, Geetanjali Chilkoti, Sunny Kumar and Ashok Kumar Saxena (2014), "Anesthetic Management of Hepatitis C Positive Patient with Portal Hypertension for Cesarean Section - A Case Report," JMED Research Vol. 2014 (2014), Article ID 773131, DOI: 10.5171/2014.773131 
bilirubin.- $2.56 \mathrm{mg} / \mathrm{dl}$, SGOT- 70IU/ml, SGPT$75 \mathrm{IU} / \mathrm{ml}$, alkaline phosphatase $320 \mathrm{IU} / \mathrm{l}$ and INR 1.6. Anti HCV antibody titre was positive. Abdominal ultrasonography and esophagogastroscopy revealed moderate hepatosplenomegaly with grade II esophageal varices (EV) with mild portal hypertension. Patient received 3 units of FFP and then was planned to be managed conservatively as advised by gastroenterologist. At 32 weeks, patient presented in emergency with two bouts of hemetemesis with minimal bleeding (approximately 10-20 $\mathrm{ml}$ ). Though, prophylactic endoscopic band ligation (EBL) was considered but could not be done as our institution lacks the facility. Patient was observed for 24 hrs and then discharged with the advice to report in case of further hemetemesis. Following this, the patient remained stable with no episodes of hemetemesis till 38 weeks when the patient presented for delivery.

On preanaesthetic evaluation, systemic examination was unremarkable and all blood investigations including haemogram, LFT, kidney function test and coagulation profile were within normal limits. Patient, at that time, did not have bleeding EV. Patient received routine aspiration prophylaxis and adequate blood was arranged preoperatively. On OT table, all routine non invasive monitors were attached and two large bore intravenous cannulaes were secured. The entire staff working in operation theatre took standard barrier precautions. Regional anesthesia was planned considering normal coagulation profile, mild portal hypertension and no actively bleeding esophageal varices. Under all aseptic precautions, subarachnoid block was instituted at L3-4 level using 26gauze, Whitacre needle and $2 \mathrm{ml}$ of $0.5 \%$ hyperbaric bupivacaine was administered in left-lateral position. After the baby was delivered, injection oxytocin 20 units were administered in slow intravenous infusion. The patient received 1.5litres of Lactated Ringer's solution and urine output was $100 \mathrm{ml}$ at the end of the surgery. Baby cried immediately after delivery and Apgar score at 1 and 5minutes was $9 / 10$ and $10 / 10$ respectively. Intraoperatively, patient remained hemodynamically stable. The patient was observed in high dependency unit for 24 hours in the postoperative period and was then transferred to ward. Patient was discharged on fifth post-operative day with the advice to have regular follow-ups in gastroenterology out patient clinic.

\section{Discussion}

In the present case report, the patient had hepatitis $\mathrm{C}$ induced portal hypertension and presented in antenatal period twice with the complaints of bleeding EV. Patient was managed conservatively and anaesthetic management for LSCS was uneventful with good maternal and fetal outcome.

According to Zeeman et al. (1999), hepatitis C affects $0.5-1.4 \%$ of obstetric population. Baig et al (2009) mentioned that the incidence of hepatitis $\mathrm{C}$ and $\mathrm{D}$ doubles with hepatitis B coinfection and combined incidence was reported to be as high as 3.1\%. Viral hepatitis during pregnancy is associated with various maternal and fetal complications like jaundice, coagulopathy, hepatic failure, bleeding from esophageal varices, hepatorenal syndrome, spontaneous abortions, premature labour and increased risk of vertical transmission. On literature search, there has been no case report of bleeding $\mathrm{EV}$ in hepatitis $\mathrm{C}$ induced portal hypertension in pregnant patient.

Pregnancy associated with viral hepatitis induced portal hypertension and esophageal varices is rare. In the book by Chestnut (2009), massive bleeding from esophageal varices has been mentioned as one of the most common causes of maternal mortality. According to Aggarwal et al, the overall risk of variceal bleeding in pregnant women with portal hypertension is almost 400 times greater than in pregnant women without portal hypertension. It may be further aggravated by deranged coagulation and thrombocytopenia. Zeeman et al have reported that the incidence of variceal 
bleeding during pregnancy reaches up to $45 \%$ with a mortality rate of $18-50 \%$. The increased incidence of variceal bleeding during pregnancy is attributed to physiological increase in circulating blood volume and elevation in portal pressure due to the compression of inferior vena cava by gravid uterus. There is no definite factor to predict variceal bleeding, but degree of portal hypertension $(>12 \mathrm{mmHg}$ ) and size of varices may be the potential predictors. Additionally, hormonal and hemodynamic changes during pregnancy may be responsible for increasing capillary fragility and venous system engorgement.

Various treatment modalities, either prophylactic or urgent endoscopic injectional sclerotherapy (EIS), endoscopic band ligation (EBL) as well as transjugular intrahepatic portosystemic shunt (TIPS) (Savage (2007)) as possible management procedures during pregnancy. EBL when done in pregnancy has the advantage of being free of the potential adverse effects related to EIS (Kochhar et al, 1990) with conventional sclerosants or pharmacological treatment. Prophylactic EBL is not an established treatment modality to prevent variceal bleeding in pregnancy. Starkel et al, Dhiman et al and Zeeman et al had successfully used prophylactic EBL in various cases to reduce the risk of bleeding EV in pregnancy. McKay and Webster in a review had mentioned that as banding acts superficially, recurrence rate with EBL is more than $30-40 \%$, more so in pregnancy, thus justifying the use of multiple attempts of EBL in various aforementioned case reports. Our patient presented twice with the complaints of bleeding $\mathrm{EV}$ in the antenatal period. Though, therapeutic EBL was definitely considered, we managed our patient conservatively considering the various factors such as grade II EV, mild portal hypertension, minimal associated bleeding, institutional limitation and evidence in favour of high recurrence rate even after EBL.

Early detection of esophageal varices during antenatal period, leads to better optimization as prophylactic/therapeutic EBL can be planned. In the textbook by Miller R D, the anaesthetic goals in bleeding EV patients include correction of coagulation, thrombocytopenia, optimization following variceal band ligation, management of ascites, maintaining adequate intavascular volume and correction of any electrolyte abnormality. In our patient, regional anaesthesia was administered as it is the preferred technique for LSCS. Owing to high risk of aspiration in view of full stomach and increased intra-abdominal pressure, regional anesthesia is definitely safer. Additionally, haemodynamic response during laryngoscopy and intubation also predisposes the parturient with esophageal varices to variceal rupture and bleeding. So, one must try to avoid any blind instrumentation of esophagus in these patients. Administration of general anesthesia, further increase the risk of maternal hypoxia, uteroplacental transfer of drugs to fetus, uterine relaxation and postpartum hemorrhage. However, if contraindications have been ruled out, regional anesthesia is the preferred technique in parturients with portal hypertension. In addition, it is also devoid of complications due to portal hypertension induced alterations in drug pharmacokinetic and pharmacodynamics i.e., altered volume of distribution due to decreased serum albumin, increased globulins, unpredictable intravascular volume and increased sensitivity to anesthetic agents.

According to Spach et al, the risk of vertical transmission ranges from 4 to $10 \%$. Ohto et al (1994), reported the risk of vertical transmission of hepatitis $C$ to be $6-10 \%$.The risk of HCV transmission to infants increases with detectable hepatitis C viremia during pregnancy. Presently, there is no safe and effective intervention which is known to prevent perinatal transmission of $\mathrm{HCV}$. Elective Cesarean section is not recommended for prevention of vertical HCV transmission. So, postoperative clinical and biochemical surveillance of neonates constitutes an important aspect of 
management. According to the Centres for Disease Control and Prevention (CDC), children should not be tested for HCV until 18 months of age, as antibodies from the mother remain in the infant's circulation till that time.

In conclusion, Hepatitis C associated portal hypertension induced bleeding esophageal varices in pregnancy is a rare presentation. Since there have been very few published case reports, the management of EV during pregnancy is still under evaluation. We would reinforce, firstly, upon the early detection of $\mathrm{EV}$ in the antenatal period so that the prophylactic/therapeutic EBL can be planned. Secondly, we must remember that the recurrence following EBL is high in pregnant patients due to the pregnancy induced physiological changes. Finally, regional anesthesia must be considered where deemed possible.

\section{References}

Aggarwal, N., Sawnhey, H., Suril, V., Vasishta, K., Jha, M. \& Dhiman, R. K.(1999). "Pregnancy and Cirrhosis of the Liver," Australian and New Zealand Journal of Obstetrics and Gynaecology, 39 (4)503-6.

Baig, S., Siddiqui, A. A., Ahmed, W. U., Qureshi, H. \& Arif, A. (2009). "Frequency of Hepatitis C and D Super Infection in Patients with Hepatitis B Related Complex Liver Disorders," Journal of the College of Physicians and Surgeons Pakistan, 19 (11) 699-703.

CDC. (1998). Centers for Disease Control and Prevention. "Recommendations for Prevention and Control of Hepatitis C Virus (HCV) Infection and HCV-Related Chronic Disease," MMWR Recommendations and Reports, 47(19) 1-39.

Chestnut, D. H., Polley, L. S., Tsen, L. C. \& Wong, C. A. (2009). Chestnut's Obstetric Anesthesia: Principles \& Practice, Ed 4
Dhiman, R. K., Biswas, R., Aggarwal, N., Sawhney, H. \& Chawla, Y. (2000). "Management of Variceal Bleeding in Pregnancy with Endoscopic Variceal Ligation and N-Butyl-2-Cyanoacrylate: Report of Three Cases," Gastrointestinal Endoscopy, 51, 91-93.

Kochhar, R., Goenka, M. K. \& Mehta, S. K. (1990). "Endoscopic Sclerotherapy during Pregnancy," American Journal of Gastroenterology 185, 1132-1135.

McKay, R. \& Webester, N. R. (2007). "Variceal Bleeding," Continuing Education in Anaesthesia, Critical Care \& Pain 7 (6) 191194.

Ohto, H., Terazawa, S., Sasaki, N. et al. (1994). "Transmission of Hepatitis C Virus from Mothers to Infants," New England Journal of Medicine, 330, 744-50.

Rothenberg, D. M., O'Connor, C. J. \& Tuman, K. J. (2010). 'Anesthesia and the Hepatobiliary System,' Miller's Anesthesia, Edn 7

Savage, C., Patel, J., Lepe, M. R., Lazarre, C. H. \& Rees, C. R. (2007). "Transjugular Intrahepatic Portosystemic Shunt Creation for Recurrent Gastrointestinal Bleeding during Pregnancy," Journal of Vascular and Interventional Radiology, 18, 902-904.

Sobral. M., Granya, C., Sampaio, M. \& Guerreiro, F. (2013). 'Bleeding from Esophageal Varices in Pregnancy,' BMJ Case Reports

Spach, D. H., Kim, H. N. \& Marrazzo, J. 'Hepatitis Web Study,' depts.washington.edu/hepstudy/hepC/preve ntion/hcvpnt/discussion.html

Starkel, P., Horsmans, Y. \& Geubel, A. (1998). "Endoscopic Band Ligation: A Safe Technique to Control Bleeding Esophageal Varices in Pregnancy," Gastrointestinal Endoscopy, 48, 212-214. 
Tan, J., Surti, B. \& Saab, S. (2008). "Pregnancy and Cirrhosis," Liver Transplantation, 14, 1081-1091

Zeeman, G. G. \& Moise, K. J. (1999).

"Prophylactic Banding of Severe Esophageal Varices with Liver Cirrhosis in Pregnancy," Obstetrics \& Gynecology 94, 842 